\title{
Article \\ Functional Translation of Exercise Responses from Exercise Testing to Exercise Training: The Test of a Model
}

\author{
Tristan Tyrrell ${ }^{1}$, Jessica Pavlock ${ }^{1}$, Susan Bramwell ${ }^{1}$, Cristina Cortis ${ }^{2}{ }^{\oplus}$, Scott T. Doberstein ${ }^{1}$, Andrea Fusco ${ }^{2}{ }^{\oplus}$, \\ John P. Porcari ${ }^{1}$ and Carl Foster ${ }^{1, *}$ \\ 1 Department of Exercise and Sport Science, University of Wisconsin-La Crosse, La Crosse, WI 54601, USA; \\ tyrrel12697@uwlax.edu (T.T.); pavlock0615@uwlax.edu (J.P.); sbramwell@uwlax.edu (S.B.); \\ sdoberstein@uwlax.edu (S.T.D.); jporcari007@gmail.com (J.P.P.) \\ 2 Department of Human Sciences, Society and Health, University of Cassino and Lazio Meridionale, \\ 03043 Cassino, Italy; c.cortis@unicas.it (C.C.); andrea.fusco@unicas.it (A.F.) \\ * Correspondence: cfosteruwl@gmail.com
}

check for

updates

Citation: Tyrrell, T.; Pavlock, J.; Bramwell, S.; Cortis, C.; Doberstein, S.T.; Fusco, A.; Porcari, J.P.; Foster, C. Functional Translation of Exercise Responses from Exercise Testing to Exercise Training: The Test of a Model. J. Funct. Morphol. Kinesiol. 2021, 6, 66. https://doi.org/10.3390/ jfmk6030066

Academic Editor: Peter Hofmann

Received: 29 June 2021

Accepted: 26 July 2021

Published: 28 July 2021

Publisher's Note: MDPI stays neutral with regard to jurisdictional claims in published maps and institutional affiliations.

Copyright: (c) 2021 by the authors. Licensee MDPI, Basel, Switzerland. This article is an open access article distributed under the terms and conditions of the Creative Commons Attribution (CC BY) license (https:// creativecommons.org/licenses/by/ $4.0 /)$.

\begin{abstract}
Exercise prescription based on exercise test results is complicated by the need to downregulate the absolute training intensity to account for cardiovascular drift in order to achieve a desired internal training load. We tested a recently developed generalized model to perform this downregulation using metabolic equivalents (METs) during exercise testing and training. A total of 20 healthy volunteers performed an exercise test to define the METs at 60,70 , and $80 \%$ of the heart rate (HR) reserve and then performed randomly ordered $30 \mathrm{~min}$ training bouts at absolute intensities predicted by the model to achieve these levels of training intensity. The training HR at 60 and 70\% HR reserve, but not $80 \%$, was significantly less than predicted from the exercise test, although the differences were small. None of the ratings of perceived exertion (RPE) values during training were significantly different than predicted. There was a strong overall correlation between predicted and observed HR $(r=0.88)$ and RPE $(r=0.52)$, with $92 \%$ of HR values within $\pm 10 \mathrm{bpm}$ and $74 \%$ of RPE values within \pm 1 au. We conclude that the generalized functional translation model is generally adequate to allow the generation of early absolute training loads that lead to desired internal training loads.
\end{abstract}

Keywords: exercise prescription; target heart rate; RPE; METs

\section{Introduction}

Exercise is a lifestyle factor that is beneficial to health [1-4]. It is beneficial on a dose-response basis, up to amounts of training of several times professional society guidelines [1,2] and only associated with health problems at levels consistent with heavy athletic training in middle-aged and older individuals [1,2,5]. In patients with chronic diseases, exercise forms a cornerstone of the treatment scheme of rehabilitation programs being of value both in terms of accelerating the rate of recovery [6], secondary prevention [7-9], and is even of value after contemporary medical care (percutaneous interventions and statins) is accounted for [10]. However, exercise training can present a significant risk of clinical events [11], particularly in the setting of unaccustomed heavy exercise in sedentary individuals [12] or in the setting of myocardial ischemia during exercise training [13]. Further, as with other behavioral interventions, new exercise programs have a disappointing pattern of compliance [14]. Although high-intensity exercise has recently become popular [15] and has well-documented efficacy [16], even in clinical populations [17-19], it is associated with at least a potentially increased risk of complications and reductions in program enjoyment and compliance [20].

In the setting of an increased risk based on age, risk factors, history of disease or symptoms, professional societies recommend graded exercise testing before beginning exercise programs, both to rule out clinically occult disease and to guide the exercise 
prescription [21]. The dominant model for subsequent exercise prescription is based on a percentage of the maximal exercise capacity (metabolic equivalents (METs)), heart rate (HR) or HR reserve (\%HRR) [21], rating of perceived exertion (RPE) [22], or ventilatory threshold (VT) [23]. Recently, the talk test, which can be taken as a surrogate of VT, has been effectively used to prescribe exercise training $[24,25]$ and to avoid ischemia during training [26].

Unfortunately, the same exercise workload during graded exercise testing (GXT) that produces a given HR, RPE, VT, or talk test response during incremental exercise, does not produce an equivalent response during sustained exercise training. The effect of cardiovascular drift dictates that the response to sustained exercise is often significantly larger than during brief stages at that same workload during a GXT. Potentially, this leads to training sessions that are harder than desired [27], which may, in turn, have adverse effects on safety and compliance. Accordingly, the exercise training workload must be reduced, downregulated, or "translated" in order to achieve desirable results during exercise training. Previous work from our laboratory has demonstrated a solution for translating exercise responses from GXT to ambulation and cycle ergometry [27,28], to arm-leg ergometry [29], to recreational activities [30]. A recent report from our laboratory demonstrated a potentially viable strategy to generalize the process of translating exercise test responses based on computing the MET cost during GXT and during training [31]. If this generalized model were shown to be accurate, then the process of translating either maximal or submaximal GXT responses to workloads useable on the first day of training would be more effective and safer. Accordingly, the intent of this study was to provide a systematic test of this generalized model on the basis of both \%HRR and RPE responses.

\section{Materials and Methods}

The subjects for this study were 20 healthy, young adult volunteers. Although all were physically active, none were systematically trained athletes. The study protocol was approved by the Institutional Review Board for the Protection of Human Subjects at the University of Wisconsin-La Crosse (protocol \# 45CFR46, approved 13 April 2021). All subjects provided written informed consent prior to participation. Characteristics of the subjects are presented in Table 1. The overall experimental approach was to start with MaxMETs, which is a normally measured variable during exercise testing, and then apply our predictive model for converting MaxMETs into the workload for exercise training [31] to test the degree to which exercise training responses (HR and RPE) during training fell within the desired range.

Table 1. Mean and standard deviation of the characteristics of the subjects.

\begin{tabular}{ccc}
\hline Characteristics & $\begin{array}{c}\text { Males } \\
(\boldsymbol{n}=\mathbf{1 0})\end{array}$ & $\begin{array}{c}\text { Females } \\
(\boldsymbol{n}=\mathbf{1 0})\end{array}$ \\
\hline Age (years) & $23.5 \pm 2.2$ & $22.3 \pm 0.9$ \\
Height $(\mathrm{cm})$ & $183.1 \pm 7.3$ & $167.6 \pm 6.7$ \\
Weight $(\mathrm{kg})$ & $82.3 \pm 18.4$ & $70.0 \pm 13.2$ \\
$\mathrm{VO}_{2}$ max $\left(\mathrm{ml} \cdot \mathrm{kg}^{-1} \cdot \mathrm{min}^{-1}\right)$ & $53.3 \pm 6.1$ & $40.3 \pm 5.3$ \\
HRmax $(\mathrm{bpm})$ & $189 \pm 7$ & $185 \pm 5$ \\
RPEmax & $19.9 \pm 0.2$ & $19.9 \pm 0.2$ \\
\hline
\end{tabular}

$\mathrm{VO}_{2}$ max: maximal oxygen uptake; HRmax: maximal heart rate; RPEmax: maximal rating of perceived exertion.

Each subject performed a GXT, to volitional fatigue, using a modified Bruce treadmill protocol [32]. During the test, HR and RPE (6-20 scale) [33] were measured at the end of each $1 \mathrm{~min}$ stage and blood pressure was measured every $3 \mathrm{~min}$. During the test, respiratory metabolism was measured using open-circuit spirometry using a mixing chamber-based metabolic system (AEI Technologies, Pittsburgh, PA, USA). Integration of gas exchange values was performed every $30 \mathrm{~s}$ and $\mathrm{VO}_{2}$ max was accepted as the highest $30 \mathrm{~s}$ value obtained during the test. The VT was computed using both the v-slope and ventilatory equivalent methods [34]. The HR reserve (HRR) was computed based on the observed 
maximal HR at the end of the test, and resting HR obtained in the standing position just before the test. The individual exercise time vs. HR curves were examined to determine the moment that the HR achieved reference values of $60 \% \mathrm{HRR}, 70 \% \mathrm{HRR}$, and $80 \% \mathrm{HRR}$, which are surrogates for easy, moderate, and hard exercise. The RPE at these moments was also noted. The speed and grade of the treadmill belt at these moments were used to calculate the MET requirement based on conventional equations [21] and are referred to as GXT METs. The training workload was computed from the generalized functional translation model [31] as $72 \%$ of the GXT METs at each of the \% HRR targets. The speed and grade required to achieve this MET level were computed by backward solving of the same equations [21]. From the solution, treadmill speeds between 1.79 and $2.23 \mathrm{~m} \cdot \mathrm{s}^{-1}$ (4-5 mph, 6.4-8 kmh) were avoided since solely walking or solely running is inconvenient in this speed range.

Each subject then performed three, randomly ordered 30 min training bouts $(5 \mathrm{~min}$ warm-up at $1.33 \mathrm{~m} \cdot \mathrm{s}^{-1}, 0 \%$ grade, $20 \mathrm{~min}$ at the targeted workload, and $5 \mathrm{~min}$ cool-down at $1.33 \mathrm{~m}^{-1}$ ). HR and RPE were measured at 5 min intervals, and the HR and RPE at $15 \mathrm{~min}, 20 \mathrm{~min}$, and $25 \mathrm{~min}$ were averaged and accepted as the HR and RPE response to the training workloads predicted by the generalized functional translation model.

The HR and RPE achieved during steady-state training at the intended easy, moderate, and hard workloads were compared to that calculated from responses during the GXT using repeated measures Analysis of variance (ANOVA). A $p$-value of $<0.05$ was accepted as statistically significant. When justified by ANOVA, pairwise comparisons were made using Tukey's test. Correlations are calculated using the Pearson product-moment correlation.

\section{Results}

The predicted and achieved HR and RPE during the last 15 min of the easy, moderate, and hard training bouts are presented in Table 2 . There were small but significant differences between predicted and achieved HR at the $60 \%$ HRR (easy) and 70\%HRR (moderate) levels of intensity. There was no significant difference between predicted and achieved $\mathrm{HR}$ at the $80 \% \mathrm{HRR}$ intensity. There were no significant differences between predicted and achieved RPE at any of the intensity levels.

Table 2. Mean and standard deviation of the comparison between the predicted and achieved values of heart rate (HR) and rate of perceived exertion (RPE) at the $60 \%, 70 \%$, and $80 \%$ of the heart rate reserve (\% HRR) intensities.

\begin{tabular}{ccccc}
\hline \% HRR & Predicted HR & Achieved HR & Predicted RPE & Achieved RPE \\
\hline $60 \%$ & $135.1 \pm 4.3$ & $130.2 \pm 7.6^{*}$ & $11.8 \pm 1.2$ & $11.2 \pm 1.5$ \\
$70 \%$ & $147.9 \pm 4.6$ & $142.8 \pm 8.9^{*}$ & $13.0 \pm 1.4$ & $12.4 \pm 1.7$ \\
$80 \%$ & $160.4 \pm 5.4$ & $157.3 \pm 9.7$ & $14.1 \pm 1.1$ & $13.8 \pm 1.3$ \\
\hline
\end{tabular}

* significantly $(p<0.05)$ different from predicted HR.

A scatterplot of predicted vs. achieved HR for the combined results of the three intensities bouts is presented in Figure 1. There did not appear to be a significant bias in the pattern of responses, and the correlations between predicted and observed HR responses for easy $(r=0.52)$, moderate $(r=0.74)$, hard $(r=0.64)$, and combined $(r=0.88)$ were uniformly strong. Overall, $92 \%$ of observed $\mathrm{HR}$ values were within $\pm 10 \mathrm{bpm}$ of the predicted values.

A scatterplot of predicted vs. achieved RPE for the combined results of the three intensities bouts is presented in Figure 2. There did not appear to be a significant bias in the pattern of responses, and the correlations between predicted and observed RPE responses for easy $(r=0.66)$, moderate $(r=0.81)$, hard $(r=0.61)$, and combined $(r=0.88)$ were uniformly strong. Overall, $74 \%$ of the observations were within the \pm 1 RPE unit of the predicted values. 


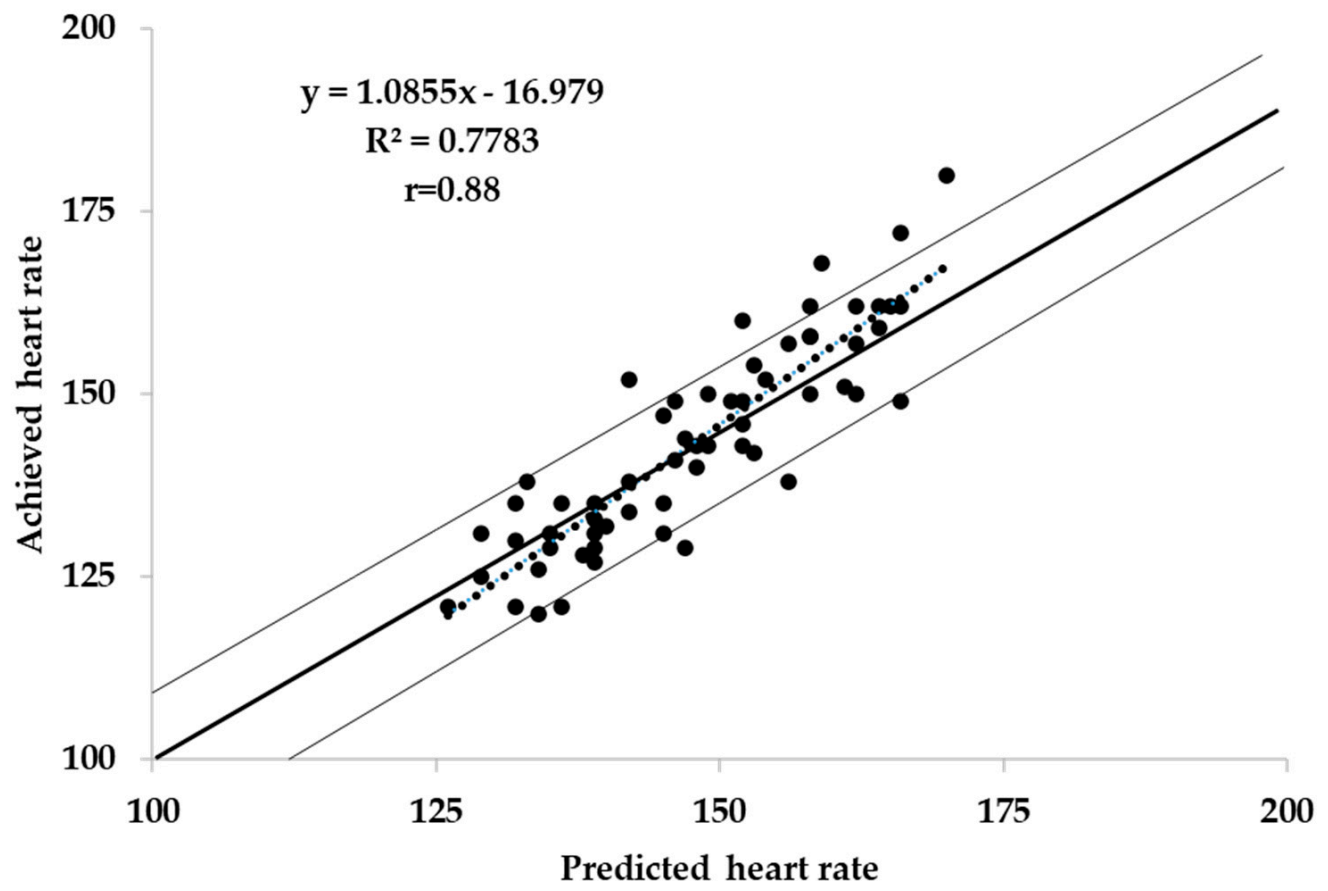

Figure 1. Predicted vs. achieved heart rate during the three intensities bouts.

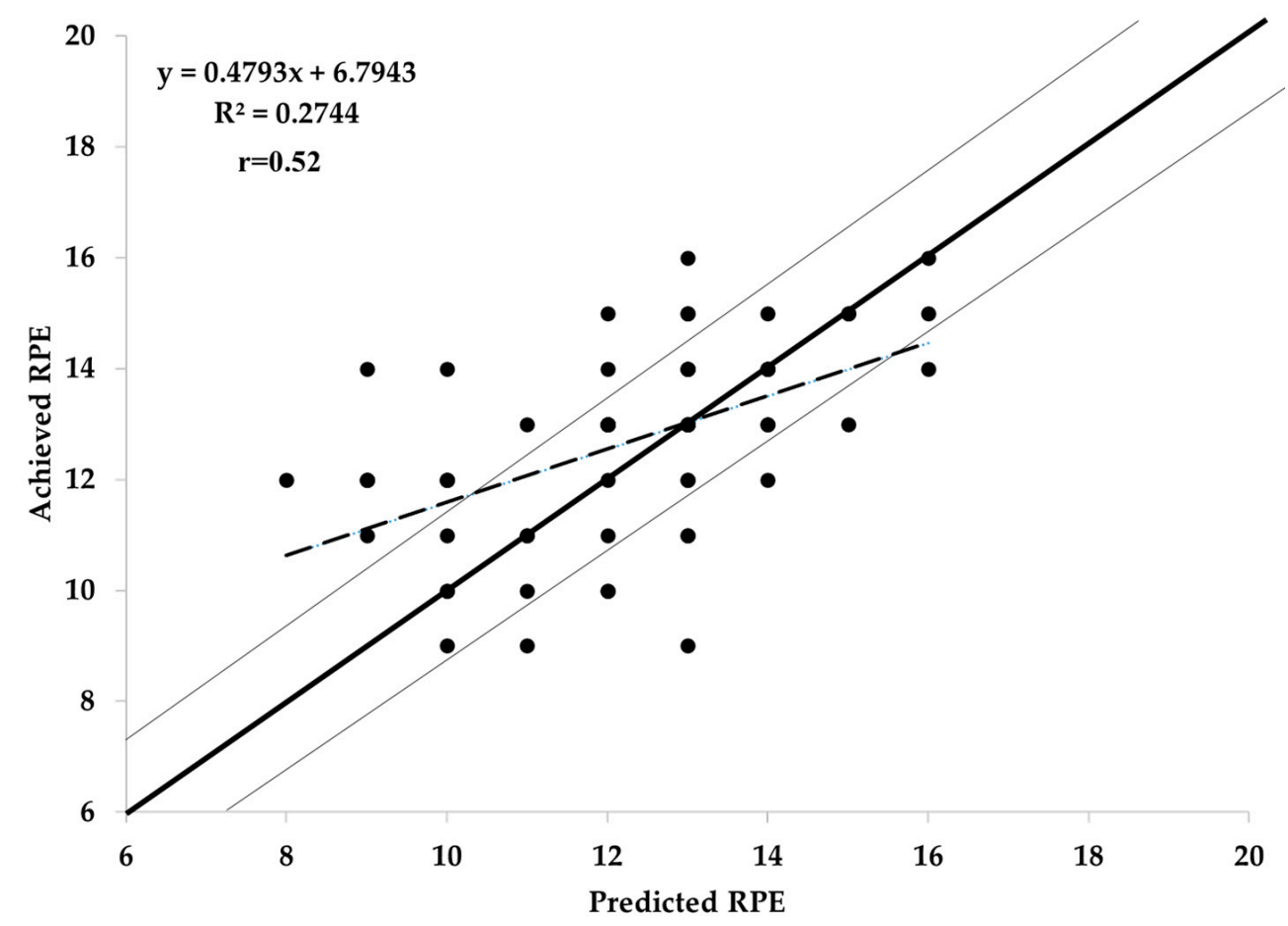

Figure 2. Predicted vs. achieved rating of perceived exertion (RPE) during the three intensities bouts.

\section{Discussion}

The main finding of this study was that the generalized model for translating GXT responses into training workloads [31] appears to be accurate over the range of training intensities commonly prescribed in fitness and rehabilitation programs. While there was a bias for the predicted HR at the 60\% (135 vs. 131) and 70\% (148 vs. 143) HRR workloads to be slightly higher than observed, $92 \%$ of observed values were within $\pm 10 \mathrm{bpm}$. Similarly, $74 \%$ of RPE values were within the \pm 1 RPE unit. Thus, the results support our 
earlier findings with more specific exercise translation approaches [27-30] but using a generalized approach.

While there was a small but significant overprediction of $\mathrm{HR}$ responses at the easy and moderate training loads, the error was small, and in a direction that would be acceptable clinically. One of the biggest concerns during exercise prescription is that workloads during the beginning days and weeks of a training program will be too heavy. This may result in reduced enjoyment [20] and may bias toward an increased likelihood of untoward events [11-13]. Thus, the tendency for the "translated" training load to be a little easier than predicted is a very acceptable error.

The primary limitation to the current results is attributable to a limitation of subject selection by the COVID-19 pandemic. In an idealized test of the generalized model, we would have selected fully sedentary individuals, or even patients from a rehabilitation program, as these are the individuals who actively need a strategy for translating GXT results into training prescriptions. However, restrictions on laboratory use dictated that younger and more active students served as the subjects. However, as none of the subjects was systematically training for sports competition, and as their $\mathrm{VO}_{2} \mathrm{max}$ spanned a considerable range of fitness, we feel that the experimental test of the generalized functional translation model remains valid.

The generalized functional translation model tested in this study was based on the results of a maximal GXT, as had been the results of the progenitor studies [27-30]. Contemporary practice in both fitness and rehabilitation communities is not to have maximal GXT results available. Where a preliminary GXT is performed, it is often submaximal in nature, limited to an RPE of 15 (hard). However, the strong relationship between the progression of RPE and relative exercise intensity $[35,36]$ and the strong relationship between the talk test and relative exercise intensity $[24,25,36]$ suggest that picking target values for RPE or the talk test from a submaximal GXT and then applying the generalized functional translation model is likely to yield the same prescriptive result as a maximal effort GXT [25].

\section{Conclusions}

The results of this study suggest that a generalized model for translating GXT results to exercise training loads, based on calculated MET values [31], yields both HR and RPE responses during training bouts that are close to predicted values. Therefore, the results suggest a prescriptive strategy based on $70-75 \%$ of the MET requirement at that level of internal training load during a GXT is likely to make the beginning portions of an exercise training program both more pleasant and safer while still being effective.

\section{Practical Applications}

As an example of the use of the functional translation model, which is validated in the present data, consider the following: A sedentary, although healthy, person performs a GXT (without handrail support) using a Balke type treadmill protocol (a constant speed with grade increments every $2 \mathrm{~min}$ ) with the results in Table 3.

Table 3. Example of a subject's speed, grade, metabolic equivalents (METs), heart rate (HR), and rating of perceived exertion (RPE) response values during a graded exercise testing using a Balke type treadmill protocol.

\begin{tabular}{ccccc}
\hline Speed $\left(\mathbf{m} \cdot \mathbf{s}^{-\mathbf{1}}\right)$ & Grade (\%) & METs [21] & HR & RPE \\
\hline 0 & 0 & 1 & 70 & 6 \\
1.34 & 0 & 3.3 & 95 & 8 \\
1.34 & 2 & 4.1 & 115 & 10 \\
1.34 & 4 & 4.9 & 133 & 12 \\
1.34 & 6 & 5.8 & 150 & 14 \\
1.34 & 8 & 6.6 & 155 & 16 \\
1.34 & 10 & 7.4 & 160 & 18.5 \\
\hline
\end{tabular}


The target HR (THR) is calculated at 70\% HRR as follows:

$$
\begin{gathered}
\mathrm{THR}=[(160-70) \times 0.7]+70 \\
\mathrm{THR}=[90 \times 0.7]+70=133 \mathrm{bpm}
\end{gathered}
$$

METs are calculated per standard equations [21] as

$$
\mathrm{VO}_{2}=\left[\left(\text { speed }\left(\mathrm{m} \cdot \mathrm{min}^{-1}\right) \times 0.1\right]+\left[\text { speed }\left(\mathrm{m} \cdot \mathrm{min}^{-1}\right) \times 1.8 \times \text { grade } / 100\right]+3.5\right.
$$

For example, at $4 \%$ grade (e.g., $70 \%$ HRR),

$$
\begin{gathered}
\mathrm{VO}_{2}=[80.4 \times 0.1]+[80.4 \times 1.8 \times 0.04]+3.5 \\
\mathrm{VO}_{2}=8.4+5.8+3.5=17.7 \mathrm{~mL} \cdot \mathrm{min}^{-1} \cdot \mathrm{kg}^{-1}=5.1 \mathrm{METs}
\end{gathered}
$$

Applying the generalized functional translation model, the training intensity becomes

$$
5.1 \times 0.72=3.7 \mathrm{METs}=13.0 \mathrm{~mL} \cdot \mathrm{min}^{-1} \mathrm{~kg}^{-1}
$$

Solving for grade at a walking speed of $1.34 \mathrm{~m} \cdot \mathrm{s}^{-1}$,

$$
\begin{aligned}
13.0= & {[80.4 \times 0.1]+[80.4 \times 1.8 \times \text { grade }]+3.5 } \\
& 13.0=8.0+[144.7 \times \text { grade }]+3.5 \\
& (13.0-8.0-3.5) / 144.7=\text { grade } \\
& 1.5 / 144.7=\text { grade }=0.01=1 \%
\end{aligned}
$$

Thus, with the tabled GXT results, one would expect $~ 70 \%$ HRR with an RPE of 11-12 at a walking speed of $1.34 \mathrm{~m} \cdot \mathrm{s}^{-1}(3.0 \mathrm{mph}$ or $4.8 \mathrm{kmh})$ at a grade of $1 \%$. While this may not provide the specific results desired, it does provide a reasonable candidate for how to prescribe the first workouts in the training facility.

Author Contributions: Conceptualization, J.P.P. and C.F.; investigation, T.T., J.P. and C.F.; data curation, T.T., J.P., J.P.P. and C.F.; writing-original draft preparation, T.T., J.P. and C.F.; writing-review and editing, T.T., J.P., S.B., C.C., S.T.D., A.F., J.P.P. and C.F.; supervision, S.B. and J.P.P. All authors have read and agreed to the published version of the manuscript.

Funding: This research received no external funding.

Institutional Review Board Statement: The study was conducted according to the guidelines of the Declaration of Helsinki and approved by the Institutional Review Board for the Protection of Human Subjects at the University of Wisconsin-La Crosse (protocol \# 45CFR46, approved 13 April 2021).

Informed Consent Statement: Informed consent was obtained from all subjects involved in the study.

Data Availability Statement: The data presented in this study are available on request from the corresponding author.

Conflicts of Interest: The authors declare no conflict of interest.

\section{References}

1. Arem, H.; Mozre, S.C.; Patel, A.; Hartge, P.; De Gonzalez, A.B.; Visvanathan, K.; Campbell, P.T.; Freedman, M.; Weiderpass, E.; Adami, H.O.; et al. Leisure time physical activity and mortality: A detailed pooled analysis of the dose-response relationship. JAMA Intern. Med. 2015, 175, 959-967. [CrossRef]

2. O'Keefe, J.; Lavie, C.J.; Guazzi, M. Potential danger of extreme endurance exercise: How much is too much? Prog. Cardiovas. Dis. 2015, 57, 396-405. [CrossRef]

3. Kraus, W.E.; Janz, K.F.; Powell, K.E.; Campbell, W.W.; Jakicic, J.M.; Troiano, R.P.; Sprow, K.; Torres, A.; Piercy, K.L. Daily step counts for measuring physical activity exposure and its relation to health. Med. Sci. Sports Exerc. 2019, 51, 1206-1212. [CrossRef]

4. Lee, I.M.; Shiroma, E.J.; Lobelo, F.; Puslza, P.; Blair, S.N.; Katzmarek, P.J. Effect of physical inactivity on major non-communicable diseases worldwide: An analysis of burden of disease and life expectany. Lancet 2012, 380, 21-27. [CrossRef] 
5. Paffenbarger, R.S.J.; Hyde, R.T.; Wing, A.L.; Hsieh, C.C. Physical activity and all-cause mortality and longevity of college alumni. N. Engl. J. Med. 1986, 314, 605-613. [CrossRef]

6. Foster, C.; Pollock, M.L.; Anholm, J.D.; Squires, R.W.; Ward, A.; Dymond, D.S.; Rod, J.L.; Saichek, R.P.; Schmidt, D.H. Work capacity and left ventricular function during rehabilitation from myocardial revascularization surgery. Circulation 1984, 69, 748-755. [CrossRef]

7. Neibauer, J.; Hambrecht, R.; Velicht, T.; Hauer, K.; Marburger, C.; Kälberer, B.; Weiss, C.; von Hodenberg, E.; Schlierf, G.; Schuler, G.; et al. Attenuate progression of coronary artery disease after 6-years of multi-factorial risk intervention. Circulation 1997, 96, 2534-2541. [CrossRef]

8. Haskell, W.; Alderman, E.; Fair, J. Intensive risk factor management in men and women with coronary artery disease. The Stanford coronary risk intervention project (SCRIP). Atherosclerosis 1984, 109, 182-189. [CrossRef]

9. O'Connor, G.T.; Buring, J.E.; Yuset, S.; O'Connor, G.T.; Buring, J.E.; Yusuf, S.; Goldhaber, S.Z.; Olmstead, E.M.; Paffenbarger, R.S., Jr.; Hennekens, C.H. An overview of randomized trials of rehabilitation with exercise after myocardial infarction. Circulation 1989, 80, 234-244. [CrossRef]

10. Rauch, B.; Davod, C.O.; Doherty, P.; Saure, D.; Metzendorf, M.I.; Salzwedel, A.; Voeller, H.; Jensen, K.; Schmid, J.P. The prognostic effect of cardiac rehabilitation in the era of acute revascularization and statin therapy: A systematic review and meta analysis of randomized and non-randomized trails. Eur. J. Prev. Cardiol. 2016, 23, 1914-1939. [CrossRef]

11. Foster, C.; Porcari, J.P.; Battista, R.A.; Udermann, B.; Wright, G.; Lucia, A. The risk of exercise training. Am. J. Lifestyle Med. 2008, 2, 279-284. [CrossRef]

12. Franklin, B.; Billezke, S. Putting the benefits and risks of aerobic exercise in perspective. Curr. Sports Med. Repts. 2012, 12, 201-208. [CrossRef]

13. Hassock, K.H.; Hartwig, R. Cardiac arrest associated with supervised cardiac rehabilitation. J. Cardiopulm. Rehabil. 1982, 2, 402-408.

14. Oldridge, N.B. Compliance and experience in primary and secondary prevention of coronary heard disease: A review. Prev. Med. $1982,12,52-70$.

15. Burgomaster, K.A.; Hughes, S.C.; Heigenhauser, G.I.F.; Bradwell, S.N.; Gibala, M.J. Six sessions of sprint interval training increases muscle oxidative potential and cycle endurance capacity in humans. J. Appl. Physiol. 2005, 98, 1985-1998. [CrossRef] [PubMed]

16. Gibala, M.J.; Little, J.P.; Macdonald, M.J.; Hawley, J.A. Physiological adaptation to low volume, high intensity interval training in health and disease. J. Physiol. 2012, 590, 1077-1084. [CrossRef]

17. Meyer, K.; Foster, C.; Georgakopoulous, N.; Hajric, R.; Westbrook, S.; Ellestad, A.; Tilman, K.; Fitzgerald, D.; Young, H.; Weinstein, H.; et al. Comparison of left ventricular function during interval and steady state exercise in patients with chronic heart failure. Am. J. Cardiol. 1998, 82, 1382-1387. [CrossRef]

18. Babraj, J.A.; Volland, N.B.J.; Keast, C.; Guppy, F.M.; Cottrell, G.; Tiernan, J.A. Extremely short duration, high intensity interval training substantially improves insulin action in young healthy males. BMC Endoc. Dis. 2009, 9, 1-8. [CrossRef]

19. Wisloff, U.; Stoylen, A.; Loeuuecker, J.P.; Bruvold, M.; Rognmo, Ø.; Haram, P.M.; Tjønna, A.E.; Helgerud, J.; Slørdahl, S.A.; Lee, S.J.; et al. Superior cardiovascular effect of aerobic interval training versus moderate continuous training in heart failure patients A randomized study. Circulation 2007, 115, 3086-3094. [CrossRef]

20. Foster, C.; Farland, C.V.; Guidotti, F.; Harbin, M.; Roberts, B.; Schuette, J.; Tuuri, A.; Doberstein, S.T.; Porcari, J.P. The effects of high intensity training vs steady state training on aerobic and anaerobic capacity. J. Sports Sci. Med. 2015, 14, 747-755.

21. ACSM. ACSM's Guidelines for Exercise Testing and Prescription, 10th ed.; Wolters Kluwer: Philadelphia, PA, USA, 2017.

22. Parfitt, G.; Evans, H.; Eston, R. Perceptually regulated training at RPE13 is pleasant and improves physical health. Med. Sci. Sports Exerc. 2012, 44, 1613-1618. [CrossRef]

23. Mezzani, A.; Hamm, L.F.; Jones, A.M.; McBride, P.E.; Moholdt, T.; Stone, J.A.; Urhausen, A.; Williams, M.A. Aerobic exercise intensity assessment and prescription in cardiac rehabilitation. J. Cardiopulm. Rehab. Prev. 2012, 32, 327-350. [CrossRef]

24. Foster, C.; Porcari, J.P.; Doro, K.; Dubiel, J.; Engen, M.; Kolman, D.; Xiong, S. Exercise prescription when there is no exercise test: The Talk Test. Kinesiology 2018, 50, 333-348.

25. Porcari, J.P.; Falck, K.; Turek, J.; Sweeney, S.; Wargowski, A.; Doberstein, S.T.; Foster, C. Comparison of \%HRR and Talk Test for exercise prescription. Kinesiology 2018, 50, 3-10. [CrossRef]

26. Cannon, C.; Foster, C.; Porcari, J.P.; Skemp-Arlt, K.M.; Fater, D.C.W.; Backes, R. The Talk Test as a measure of exertional ischemia. Am. J. Med. Sport 2004, 6, 52-57.

27. Foster, C.; Porcari, J.P.; Gibson, M.; Wright, G.; Greany, J.; Talati, N.; Recalde, P. Translation of submaximal exercise test responses to exercise prescription using the Talk Test. J. Str. Cond. Res. 2009, 23, 2425-2429. [CrossRef]

28. Foster, C.; Lemberger, K.; Thompson, N.N.; Sennett, S.M.; Hare, J.; Pollock, M.L.; Pels, A.E., III; Schmidt, D.H. Functional translation of exercise responses from graded exercise testing to exercise training. Am. Heart J. 1986, 112, 1309-1316. [CrossRef]

29. Foster, C.; Thompson, N.N.; Bales, S. Functional translation of exercise responses using combined arm and leg ergometery. Cardiology 1991, 78, 150-155. [CrossRef] [PubMed]

30. Foster, C.; Thompson, N.N. Functional translation of exercise test responses to recreational activities. J. Cardiopulm. Rehabil. 1991, 11, 373-377. [CrossRef]

31. Foster, C.; Anholm, J.D.; Bok, D.; Boullosa, D.; Condello, G.; Cortis, C.; Fusco, A.; Jaime, S.J.; de Koning, J.J.; Lucia, A.; et al. Generalized approach to translating exercise tests and prescribing exercise. J. Funct. Morph. Kin. 2020, 5, 63. [CrossRef] 
32. Foster, C.; Porcari, J.P.; Hipp, M. Clinical exercise testing. In ACSM's Guidebook for Clinical Exercise Physiologists; Thompson, W., Ed.; Wolters Kluwer: Baltimore, MD, USA, 2019; pp. 349-364.

33. Borg, G.A.V. Borg's Perceived Exertion and Pain Scales; Human Kinetics Press: Champaign, IL, USA, 1998.

34. Foster, C.; Cotter, H.M. Blood lactate, respiratory and heart rate markers on the capacity for sustained exercise. In Physiological Assessment of Human Fitness, 2nd ed.; Maud, P.J., Foster, C., Eds.; Human Kinetics Press: Champaign, IL, USA, 2005; pp. 63-76.

35. Eston, R.; Lambrecht, D.; Shephard, K.; Parfitt, G. Prediction of maximal oxygen uptake from a perceptually regulated submaximal graded exercise test. J. Sports Sci. 2008, 26, 131-139. [CrossRef] [PubMed]

36. Alamji, R.A.; Foster, C.; Porcari, J.P.; Radtke, K.; Doberstein, S. Comparison of non-maximal tests for estimating exercise capacity. Kinesiology 2020, 52, 10-18. 